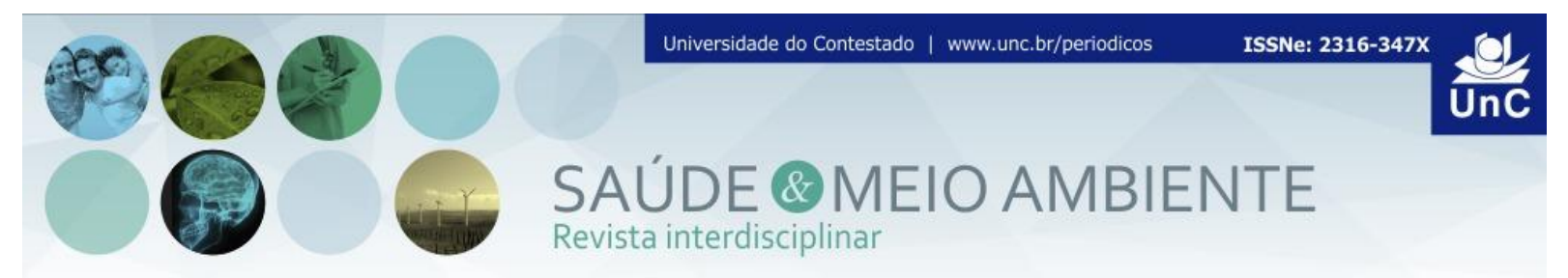

\title{
ENTRAVES À EFETIVAÇÃO DE POLÍTICAS PÚBLICAS DE SAÚDE NA RESERVA EXTRATIVISTA RIO XINGU
}

\author{
Sidney Fortunato da Silva Junior ${ }^{1}$ \\ Vinicius Campos Paraense ${ }^{2}$ \\ Tais Silva de Jesus ${ }^{3}$
}

\begin{abstract}
RESUMO
O modelo de saúde oficializado pelo Estado não é universal, mas se propõe a ser. E nessa tentativa, depara-se com a dificuldade de considerar os aspectos socioambientais e culturais na formulação e implementação de políticas públicas de saúde destinadas às populações do meio rural. Portanto, o presente trabalho se propôs a avaliar os entraves à efetivação dos serviços públicos de saúde na RESEX Rio Xingu, por meio da percepção de cinco organizações socioambientais e de saúde. O estudo apresentou convergência de opiniões em relação à insuficiência de financiamento e à inadequação das políticas publicas de saúde à realidade da RESEX. Constatou-se que os financiamentos de programas de saúde promovidos pela empresa Norte Energia e pelo Plano de Desenvolvimento Regional Sustentável do Xingu (PDRSX) podem servir de moldes para estruturação de outras políticas públicas. E por último, as organizações destacaram a importância da socialização do poder através do estreitamento das relações entre os moradores da RESEX e instituições estatais e governamentais, fomentando a participação destes em conferencias de saúde, audiências públicas e outros espaços de consulta popular.
\end{abstract}

Palavras-Chave: RESEX. Saúde pública. Desenvolvimento rural.

\section{BARRIERS TO HEALTH PUBLIC POLICY IIMPLEMENTATION IN EXTRACTIVE RESERVE RIO XINGU}

\begin{abstract}
The health model made official by the state is not universal. However, it intends to be, and in its attempt, it has faced with the difficulty of considering the social, environmental and cultural aspects in the formulation and implementation of public health policies for the people of rural areas. Therefore, this study aimed to assess the obstacles to the implementation of public health services in the extractive reserve

\footnotetext{
${ }^{1}$ Graduação em Engenharia Florestal pela Universidade Federal do Pará, Brasil. Monitor de Projetos do Instituto Avaliação, Pesquisa Programas e Projetos Socioambientais. Brasil. E-mail: sidneyfir@hotmail.com

${ }^{2}$ Mestrado em Economia pela Universidade da Amazônia, Brasil. Professor da Universidade Federal do Pará, Brasil. E-mail: viniciuscp@ufpa.br

${ }^{3}$ Graduação em Ciências Biológicas pela Universidade Federal do Pará, Brasil. E-mail: dejesus.tais@hotmail.com
} 
(RESEX) called Rio Xingu, through the perception of five environmental organizations, and health ones. The study showed agreement among opinions concerning to insufficient funding, and inadequate public health policies to the reality of RESEX. It was found that the health programs funded by the company Norte Energia and by the Sustainable Xingu Regional Development Project (PDRSX) can serve as patterns for structuring other public policies. Finally, the organizations highlighted the importance of power socialization by narrowing the relations between the inhabitants of RESEX and state and government institutions, fostering their participation in health conferences, public hearings and other public consultation spaces.

Keywords: RESEX. Public health. Rural development.

\section{INTRODUÇÃO}

Devido os intensos processos de degradação ambiental que a região amazônica tem passado em consequência de sua colonização, o poder público tem direcionado alternativas sustentáveis para o uso racional dos recursos naturais, por meio da coordenação harmoniosa dos aspectos sociais, ambientais e econômicos (PARAENSE, 2013)

Por conta disso, houve no último período a intensificação da criação de Reservas Extrativistas (RESEX), Florestas Nacionais (FLONA), Planos de Manejo Florestal Sustentável (PMFS), entre outros, aumentando assim a demanda pelo desenvolvimento de técnicas, métodos e programas de auxílio a essas áreas (RODRIGUES, 2010).

A região que compreende a Terra do Meio é muito rica em biodiversidade e se constitui em um dos maiores mosaicos de áreas de conservação do mundo (CASTRO, 2013). Nessa região encontram-se muitas Unidades de Conservação Federais e Estaduais (UC), como Terras Indígenas (TI), um Parque Nacional, uma Estação Ecológica (ESEC), uma Floresta Nacional (FLONA), uma Floresta Estadual (FLOTA), uma Área de Proteção Ambiental Estadual (APA) e três RESEX (Riozinho do Anfrisio, Rio Iriri e Rio Xingu).

As RESEX são áreas da União voltadas à proteção dos meios de vida dessas populações e conservação da biodiversidade e manutenção de um conjunto de serviços socioambientais fundamentais para sustentabilidade ecológica da Amazônia. As populações que lá residem tem um papel fundamental na manutenção desses serviços e merecem uma atenção diferenciada por parte do Estado, considerando as especificidades culturais e regionais e as adversidades das suas condições de vida (BRASIL, 2000; CASTRO, 2013; INSTITUTO SOCIOAMBIENTAL, 2013).

Dentre os vários problemas estruturantes encontrados na RESEX Rio Xingu, a saúde tem sido apontada como um problema antigo, destacada pelo Plano de 
Manejo como principal fator a ser enfrentado para garantir a qualidade de vida dos extrativistas.

No Plano de Manejo da RESEX Rio Xingu, o Subprograma de Saúde e Segurança Alimentar alerta à precariedade da situação da saúde, visto que os moradores não têm acesso ao atendimento médico básico, tampouco especializado, nem a programas de prevenção de doenças, e também não recebem ajuda dos órgãos competentes para o translado dos pacientes até a cidade, buscando o atendimento nos postos de saúde das aldeias indígenas, ou até mesmo à espera da passagem de regatões e outras embarcação a caminho da cidade (PLANO DE MANEJO DA RESEX RIO XINGU, 2012).

A Secretaria Municipal de Saúde de Altamira é a principal fonte de atendimento às necessidades de serviços de saúde na reserva. No entanto, a rede de serviços em saúde de Altamira aponta dificuldades da gestão municipal para oferecer serviços de qualidade tanto à sede do município quanto ao meio rural. Tais dificuldades são ainda maiores em regiões que apresentam isolamento, dispersão populacional, diferenças culturais, e as especificidades próprias da RESEX em questão.

Por outro lado, temos um Sistema Único de Saúde oficializado pelo estado que busca se universalizar às particularidades de cada território (BRASIL, 1988), e nesse processo surge a necessidade de políticas públicas diferenciadas para as regiões mais isoladas dos centros urbanos. A universalidade do acesso aos serviços públicos em todos os níveis de assistência está escrito na Lei 8.080/90, que cria as principais diretrizes da política nacional de seguridade social.

Portanto, este trabalho tem o propósito de reunir os principais entraves encontrados nas implementações de políticas públicas de saúde na RESEX Rio Xingu, a partir da percepção de cinco instituições: Instituto Socioambiental (ISA); Secretaria Municipal de Saúde de Altamira (SEMSA); Fundação Viver Produzir e Preservar (FVPP); Instituto Chico Mendes de Conservação da Biodiversidade (ICMBio); e Associação de Moradores do Médio Xingu (AMOMEX), visando subsidiar o fomento de futuras ações em saúde e dar visibilidade às dificuldades que inviabilizam a efetividade destas.

\section{MATERIAL E MÉTODOS}

\section{ÁREA DE ESTUDO}

A região que compreende a Terra do Meio é muito rica em biodiversidade e se constitui em um dos maiores mosaicos de áreas de conservação do mundo. Nessa região encontram-se muitas Unidades de Conservação Federais e Estaduais (VASCONCELOS et al., 2008). A Terra do Meio é assim chamada por estar 
localizada no "meio" do estado do Pará na área de interflúvio dos rios Xingu e Tapajós (CASTRO, 2013).

A Reserva Extrativista Rio Xingu Possui uma área de aproximadamente 300.000 hectares, localizada totalmente no município de Altamira - PA, a população é formada basicamente por extrativistas que englobam pescadores, seringueiros, ribeirinhos, entre outros (INSTITUTO SOCIAMBIENTAL, 2015).

Segundo Castro (2013), o número de famílias cadastradas na RESEX era de 56 no total, que se distribuem geograficamente em 11 comunidades, sendo as 4 mais povoadas: Baliza, Pedra Preta, Morro Grande e Morro do Félix. Estas comunidades encontram-se distantes uma da outra, a exemplo da Baliza e Morro do Félix, localizadas no extremo norte e no extremo sul da RESEX.

\section{COLETA E ANÁLISE DE DADOS}

Os dados foram obtidos nos meses de junho e julho de 2015, com a realização de entrevistas norteadas por roteiros semiestruturados, tendo o objetivo de: fazer com que as instituições abordassem quais eram as suas relações com a implementação de políticas públicas de saúde dentro da RESEX Rio Xingu; identificar os principais entraves que dificultam ou inviabilizam a efetividade dessas políticas; e apontar as possíveis soluções aos entraves encontrados durante suas ações em saúde.

As entrevistas foram realizadas na sede do município de Altamira - PA, o que fez com que a necessidade de avaliação da pesquisa por comitê de ética fosse dispensada, e foram conduzidas por dois pesquisadores, onde um realizou o diálogo extrativo dos dados, e o outro sistematizou as informações pertinentes ao objeto da pesquisa. Quando autorizado pelos entrevistados, as conversas foram gravadas com o auxílio de um gravador de áudio digital e, posteriormente, utilizadas para ajustar as informações escritas.

A partir da sistematização do conteúdo das entrevistas, realizou-se o feedback dos dados junto aos entrevistados, onde os mesmos puderam sugerir alterações ou complementações ao trabalho, visando a ratificação das informações divulgadas.

A entrevista realizada com representante da Associação de Moradores da RESEX Rio Xingu contou com questões adicionais, considerando o potencial das informações coletadas diretamente das pessoas que vivem na RESEX, e utilizando o método de análise de conteúdo proposto por Chizzotti (2003), que entende que tanto a coleta de dados quanto a sistematização das informações devem ser orientadas por questões que o trabalho necessita responder, denominada pelo autor de "entrevista livre semiestruturada", onde o informante discursa sobre o assunto da forma que quiser e é orientado apenas por palavras-chave, diminuindo a estranheza 
entre os termos técnicos dos questionários convencionais e as especificidades socioeconômicas e culturais dos entrevistados.

\section{RESULTADOS E DISCUSSÃO}

\section{CARACTERIZAÇÃO DAS ORGANIZAÇÕES ENTREVISTADAS}

-Secretaria Municipal de Saúde de Altamira - SEMSA: é a principal financiadora das ações e projetos em saúde para os povos da Terra do Meio. Tem atuado no sentido de fortalecer a atenção básica em saúde na RESEX com o intuito de diminuir a necessidade de deslocamento até a cidade, focando no atendimento primário, realizações de comandos médicos ${ }^{4}$, e ações de vigilância.

-Fundação Viver Produzir e Preservar - FVPP: organização sem fins lucrativos, não atua diretamente no fomento à elaboração e execução de políticas públicas de saúde na RESEX Rio Xingu, mas age no empoderamento e organização das famílias desde 1991, quando foi criada por camponeses e pastorais da Igreja Católica. Presta apoio no translado dos ribeirinhos para atendimento médico na cidade e age em parceria às ações realizadas na Terra do Meio, principalmente em saúde e educação. Não obstante, realiza projetos com a captação externa de recursos e auxilia a execução de projetos de outras organizações.

- Instituto Chico Mendes de Conservação da Biodiversidade - ICMBio: uma autarquia da União com o objetivo de propor, gerir, proteger, fiscalizar e monitorar todas as ações e programas que envolvem a RESEX Rio Xingu através da dedicação exclusiva de um servidor público que trabalha como gestor.

-Instituto Socioambiental - ISA: Organização da Sociedade Civil de Interesse Público (OSCIP) e tem o objetivo de propor soluções de forma integrada para questões sociais e ambientais para a garantia dos direitos humanos e direitos sociais, coletivos e difusos relativos ao meio ambiente. Atua fortemente no monitoramento de indicadores socioambientais na RESEX e fomenta a pressão judicial e governamental para o alcance de políticas públicas para os povos, principalmente na produção sustentável, saúde e educação.

-Associação de Moradores do Médio Xingu - AMOMEX: é a entidade que representa os moradores da RESEX Rio Xingu, sendo responsável pela

\footnotetext{
${ }^{4}$ Ações conjuntas realizadas anualmente onde algumas especialidades, na área de odontologia,
} vacinas e diagnóstico e prevenção de malária são realizadas. 
realização dos estudos iniciais à criação do Plano de Manejo da RESEX Rio Xingu.

\section{A LUTA PELO DIREITO À SAÚDE}

As populações da Terra do Meio, antes da criação de suas Reservas Extrativistas, viveram historicamente à margem da exclusão social devido ao total abandono do Estado, tiveram então que produzir e acumular conhecimento no sentido de superar as deficiências geradas pela ausência estatal e falta de políticas públicas, saber este transmitido e melhorado ao longo de gerações.

O conhecimento tradicional destes povos, aliado às características dos rios, dos animais, da floresta e de sua cultura, sempre foi essencial ao cuidado com a saúde, onde anciões experientes exercem a função de profissionais de saúde, e a biodiversidade nativa funciona como farmácia.

Com a necessidade de proteger os recursos naturais e o modo de vida da população tradicional, ameaçados pelo intenso desmatamento ilegal, iniciou-se o processo de criação das RESEX da Terra do Meio, sendo aprovado o Plano de Manejo da RESEX Rio Xingu no ano de 2012, sendo ponto de partida das ações estatais no âmbito de promover políticas públicas na região.

No entanto, os Planos de Manejo das RESEX foram criados com pouca participação dos extrativistas e, como consequência, os saberes tradicionais, não apenas no âmbito da saúde, mas também em relação à produção, educação e aspectos culturais, não foram considerados durante a elaboração (LIMA, 2015; CASTRO, 2013), visto que para Diegues (1994, p.90):

\footnotetext{
Reconhecer a importância do saber tradicional é, implicitamente, reconhecer que as população tradicionais são sujeitos históricos, portadores desse conhecimento e que, portanto, devem ser considerados como agentes fundamentais no conhecimento e sobretudo na gestão ou manejo dessas áreas chamadas naturais. Esse reconhecimento poderia retirar uma parte do poder conferido pela ciência às corporações tecnoburocraticas e acadêmicas que se autoconferem a exclusividade do saber cientifico e dos princípios e metodologias que regem os chamados "planos de manejo" das áreas naturais protegidas.
}

Em 2010, o ISA, com o apoio de organizações parceiras, realizou um diagnóstico situacional da saúde nas três RESEX da Terra do Meio, cuja proposta foi criar um plano de gestão em saúde local, através do levantamento das principais demandas apontadas pelos extrativistas e técnicos das entidades envolvidas, dando origem ao chamado "Modelo de Atenção Integral à Saúde das comunidades extrativistas da Terra do Meio", que serviu como um marco importante na luta pelo acesso à saúde, à medida que este serviu para dar visibilidade à precariedade da saúde e à ausência estatal na área, sendo um importante instrumento de pressão para autoridades competentes. 
Diante disso, os moradores das RESEX, através de suas associações, acionaram o Ministério Público Federal (MPF) acerca da violação de direitos em relação à saúde. E no ano de 2013, o MPF enviou uma procuradora da República à área, a fim de melhor entender a realidade da saúde nessas comunidades.

Durante a estada da procuradora, uma criança de 13 anos foi picada por escorpião e faleceu no local devido à falta de atendimento, pois o posto de saúde da RESEX não estava funcionando na ocasião, e a assistência médica mais próxima estava a dois dias de barco, na sede do município de Altamira, o que provocou grande repercussão na mídia que destacava "morte de menino expõe isolamento de beiradeiros" (BBC-Brasil, 2013) e, com isso, a procuradora viabilizou o estreitamento dos extrativistas e com o MPF, por meio da realização de audiências públicas com as autoridades competentes.

Nesse sentido, o Ministério da Saúde ajustou o repasse de recursos às comunidades isoladas e, assim, a criação da Lei $3.187 / 13$ pela Prefeitura de Altamira, garantindo a criação de cargos de enfermeiros e técnicos de enfermagem para atuarem na região da Terra do Meio, com remunerações diferenciadas diante da especificidade da área.

\section{A PERCEPÇÃO DAS ORGANIZAÇÕES ACERCA DOS DESAFIOS NA IMPLEMENTAÇÃO DE POLIITICAS PÚBLICAS DE SAÚDE}

As entrevistas demonstraram grande convergência de opiniões, que apontam a insuficiência de financiamento e a inadequação das políticas do Ministério da Saúde à realidade da Terra do Meio, como sendo os principais desafios à formulação e implementação de políticas públicas de saúde na RESEX Rio Xingu.

Devido aos poucos recursos que a SEMSA possui para realizar ações de atenção básica à saúde primária e subsidiar alguns serviços e insumos dentro dos Postos de Saúde das RESEX, dentre eles o do Gabirú ${ }^{5}$, nem todos os atendimentos podem ser realizados no local, como por exemplo, o tratamento de hipertensão e diabetes, que são encaminhados ao Hospital Municipal São Rafael em Altamira.

A deficiência na atenção básica à saúde na RESEX tem transformado casos menos graves em casos de média e alta complexidade, diante da falta de atendimento, aumentando a demanda por leitos, já insuficientes, do Hospital Regional Público da Transamazônica ${ }^{6}$, único hospital de referência na região para tratamento de quadros graves.

O maior gargalo encontrado para o subsídio de ações que ampliem a atenção básica na RESEX Rio Xingu é a carência de financiamentos, onde a portaria 2.488

\footnotetext{
${ }^{5}$ Localidade da RESEX Rio Xingu onde localiza-se o Posto de Saúde.

${ }^{6}$ Único hospital público para tratamento de casos de alta e média complexidade na região, que segundo a SEMSA, já apresenta um número insuficiente de leitos para as demandas das áreas urbanas dos municípios geridos pela Regional da SESPA.
} 
de 21 de Outubro de 2011 do Ministério da Saúde, que versa sobre a destinação de recursos para atenção primária em saúde de ribeirinhos, revela-se a única reconhecida pelas organizações. Para a SEMSA e ISA, a principal dificuldade em acessar fontes externas para o financiamento das ações é a falta de adequação que as atuais políticas públicas de saúde têm à realidade:

O Ministério da Saúde lançou uma portaria que trata sobre o Programa de Saúde Ribeirinho, que para nós ele não se encaixa, pois esse financiamento é para aquisição de embarcações, e o porte delas é muito grande para navegabilidade no Rio Xingu, elas navegariam durante 6 meses e o restante do ano ela ficaria parada. Então falta uma adaptação das políticas públicas à realidade das RESEX (informação verbal) ${ }^{7}$.

Existem fontes de financiamento privado que podem servir de modelo para elaboração de políticas públicas de saúde moldadas às especificidades das RESEX. Recursos estes, provindos da Norte Energia ${ }^{8}$, que financiam ações e projetos no sentido de minimizar os grandes índices de ocorrência de malária, por meio de uma condicionante específica imposta pelo IBAMA durante o processo de licenciamento da Hidrelétrica de Belo Monte, e também do Projeto de Desenvolvimento Regional Sustentável do Xingu - PDRSX através da aprovação do projeto "Assistência em Saúde às Populações Ribeirinhas das Reservas Extrativistas da Terra do Meio" submetido pela SEMSA.

Houve uma grande redução nos índices de malária naquela região, principalmente por que elas obtiveram uma atenção especial em termos de financiamento da Norte Energia, onde cerca de mais de 30 milhões de reais foram investidos e pensados especificamente e por uma equipe especializada no assunto. Hoje em dia um dos problemas que antes eram parte do cotidiano das RESEXs, foi quase que erradicado na totalidade. (informação verbal) $^{9}$

A experiência de financiamento específico para o caso da malária poderia servir de moldes para que outras políticas fossem efetivadas atendendo as características ambientais e culturais desses povos.

As organizações entrevistadas, com exceção da SEMSA e ICMBio, declararam não possuir ação direta na elaboração de políticas públicas de saúde para a RESEX Rio Xingu, mas atuam na acumulação de recursos via financiamento externo e também no empoderamento e organização das famílias para conquistas dos direitos fundamentais.

Outro entrave encontrado por este estudo reside no fato de que financiamento das ações em saúde para e região da Terra do Meio não precisa apenas ser ampliado, mas também diferenciado, considerando a participação efetiva dos extrativistas na elaboração destas.

\footnotetext{
${ }^{7}$ Informação obtida em entrevista com representante da Secretaria Municipal de Saúde de Altamira.

${ }^{8}$ Empresa responsável pela construção da Usina Hidrelétrica de Belo Monte, que foi responsabilizada pelo IBAMA de cumprir condicionantes para obter as etapas de licenciamento ambiental da obra, uma dessas condicionantes trata sobre a implementação de um Plano de Ação e Controle da Malária (PACM-Belo Monte).

${ }^{9}$ Informação obtida em entrevista com representante da Fundação Viver Produzir e Preservar.
} 


\begin{abstract}
A gente acredita que o principal desafio não é apenas o financiamento das ações, é preciso diferenciar. E a outra é os governos, todos eles, assumirem um olhar para essas comunidades não apenas como despesa, mas como fonte de desenvolvimento, e que já contribuem com o desenvolvimento do país, enquanto os governos tiverem a visão de que essas comunidades são um peso, pois se não fossem os ribeirinhos e os indígenas nós não teríamos mais nenhuma riqueza dessas que temos aí. Enquanto elas forem tratadas como peso e não como sujeitos de direitos, nós teremos estes problemas (informação verbal). ${ }^{10}$
\end{abstract}

Contudo as entrevistas às organizações demonstraram uma baixa participação dos moradores da RESEX Rio Xingu em fóruns de discussão sobre saúde, e pouca consulta direta junto à comunidade. Não obstante, uma importante conquista foi alcançada com a participação de delegados da Terra do Meio na Conferência Municipal de Saúde, evento de consulta popular para fomento e elaboração de ações de saúde em todas as esferas governamentais.

Por último, a AMOMEX destaca como desafio em curto prazo, a elaboração de um fluxograma de atendimento diferenciado para os extrativistas que procuram a cidade para obter atendimento nas unidades de saúde da sede do município, visando diminuir à demora no atendimento.

\title{
CONSIDERAÇÕES FINAIS
}

O conhecimento tradicional dos povos da Terra do Meio sempre foi primordial ao seu desenvolvimento e, principalmente, à evolução de técnicas empíricas destinadas à saúde. Porém, a ineficiência dos serviços públicos de saúde tem deixado as populações tradicionais, assim como os moradores da RESEX Rio Xingu, fora do alcance das políticas de saúde.

No entanto, a presença de organizações não governamentais, como a FVPP e o ISA, a partir da elaboração do Plano de Manejo, demonstrou grande influência no início do processo de organização e controle social dos moradores da RESEX, para a pressão das autoridades governamentais à garantia dos direitos fundamentais de saúde.

Para garantir a universalidade de acesso aos serviços públicos de saúde, de acordo com a Lei 8.080/90, é necessário que estes sejam adaptados à realidade de seus usuários. No caso da RESEX Rio Xingu, o financiamento das ações e projetos de saúde pública precisa atender às especificidades culturais e ambientais deste território, bem como criação de mecanismos de participação popular para que as demandas em saúde possam sejam apontadas e, assim, diagnosticadas pelas organizações e poder público, sob o protagonismo dos próprios moradores.

É importante ter como moldes outras experiências de financiamento e gestão de políticas públicas, tais como os modelos da Norte Energia e PDRSX, que podem

${ }^{10}$ Informação obtida em entrevista com representante da Fundação Viver Produzir e Preservar 
corroborar ao processo de elaboração de políticas em saúde. E que haja um registro das alternativas construídas pelos extrativistas na superação das suas próprias dificuldades.

\section{REFERENCIAS}

BRASIL. Constituição (1988). Constituição [da] Republica Federativa do Brasil. Brasília, DF: Senado Federal.

BRASIL. Lei no. 8.080, de 19 de setembro de 1990. Dispõe sobre as condições para a promoção, proteção e recuperação da saúde, a organização e o funcionamento dos serviços correspondentes e dá outras providências. Diário Oficial da União, Brasília, DF. 1990a. Disponível em: <http://www.planalto.gov.br/ccivil_03/ leis//8080.htm>. Acesso em: 22 set. 2015

BRASIL. Lei no. 8.142, de 28 de dezembro de 1990. Dispõe sobre a participação da comunidade na gestão do Sistema Único de Saúde (SUS) e sobre as transferências intergovernamentais de recursos financeiros na área da saúde. Diário Oficial da União, Brasília, DF. 1990b. Disponível em: <http://www.planalto.gov.br/ccivil_03/ leis//8142.htm>. Acesso em: 22 set. 2015.

BRASIL. Lei no. 9.985, de 18 de julho de 2000. Institui o Sistema Nacional de Unidades de Conservação. Diário Oficial União, Brasília, DF. 2000. Disponível em: <http://www.planalto.gov.br/ccivil_03/Leis/L9985.htm>. Acesso em: 29 set. 2015.

CASTRO, R. R. A. Comunidades Tradicionais e Unidades de Conservação no Pará: A influência da criação da Reserva Extrativista Rio Xingu - Terra do Meio, nos modos de vida das famílias locais. 2013. 167 f. Dissertação (Mestrado) - Curso de Programa de Pós-graduação em Agriculturas Amazônicas, Universidade Federal do Pará, Belém, 2013. Disponível em:

<http://mafds.websimples.info/files/arquivo/156/roberta-rowsy.pdf>. Acesso em: 27 mar. 2015.

CHIZZOTTI, A. Pesquisa em ciências humanas e sociais. 6. ed. São Paulo: Cortez, 2003. (Biblioteca da educação. Série 1. Escola; v. 16).

DIEGUES, A. C. S. O Mito Moderno da Natureza Intocada. São Paulo: Nupaub, 1994.

INSTITUTO SOCIAMBIENTAL (Org.). RESEX Rio Xingu. Disponível em: <http://uc.socioambiental.org/uc/5803>. Acesso em: 27 mar. 2015.

INSTITUTO SOCIOAMBIENTAL (Brasil) (Org.). Modelo de Atenção Integral à Saúde: e das comunidades extrativistas isoladas geograficamente da Terra do Meio. Altamira, 2013. 


\section{LIMA, F. F. $O$ acesso as políticas públicas por populações tradicionais e a} gestão de unidades de conservação de uso sustentável: o caso das reservas extrativistas da Terra do Meio, Altamira/PA. 2015. 115 f. Dissertação (Mestrado). Instituto Nacional de Pesquisas Amazônicas - Manaus. 2015.

PARAENSE, V. C.; MENDES, F. A. T.; FREITAS, A. D. Avaliação econômica de sistemas agroflorestais de cacau e mogno na transamazônica: um estudo de caso. ENCICLOPÉDIA BIOSFERA, Centro Científico Conhecer - Goiânia, v. 9, n.16; 11 p. 2013.

PLANO DE MANEJO DA RESEX RIO XINGU, 2012. Aprovado pela Portaria № 62, de 18 de maio de 2012 e publicado no Diário Oficial da União, 21 maio 2012. Disponível em: <http://www.icmbio.gov.br/portal/images/stories/imgs-unidadescoservacao/PM-RESEX-Rio-Xingu-2012.pdf>. Acesso em: 27 mar. 2015.

RODRIGUES, D. Condições de saúde dos moradores das reservas extrativistas do riozinho do Anfrísio e do rio Iriri e subsídios para a organização de serviços de saúde para essas comunidades. São Paulo. Relatório de Consultoria, 2010. Instituto Socioambiental.

VASCONCELOS, V. O.; SIQUEIRA, C. T. Populações ribeirinhas da Amazônia e preservação da cultura tradicional: dilemas em uma sociedade globalizada, 2008.

Artigo recebido em: 06/10/2015

Artigo aprovado em: 12/01/2016 